\title{
Deformation and Damage Mechanisms of Novel Austenitic ODS Steel Under In Situ ACOM-TEM Straining
}

\author{
Ankur Chauhan $^{1}$, Dimitri Litvinov ${ }^{1}$, Benjamin Kaiser $^{1}$, Jarir Aktaa $^{1}$ \\ 1. Karlsruhe Institute of Technology (KIT), Institute for Applied Materials (IAM), Eggenstein- \\ Leopoldshafen, Germany.
}

Oxide dispersion strengthened (ODS) steels are the promising candidate structural materials for future fusion or enhanced fission reactors due to their superior high temperature strength and creep resistance in comparison to their non-ODS counterpart. In this work, in situ straining inside a transmission electron microscope (TEM) was applied to directly investigate the deformation and damage mechanisms in novel austenitic ODS steel [1]. Imaging and quantification of the microstructure under straining is performed with the help of automated crystal orientation mapping (ACOM) using precession electron diffraction in scanning TEM (STEM) mode [2].

The orientation maps obtained from the same area before and after tensile straining at room temperature are shown in Figure 1a-b. From Figure 1a, it is clear that in undeformed/as-received state the steel exhibits almost equiaxed grains with no preferential texture. In addition, some grains exhibit typical crystallographic defects such as stacking faults, twins and dislocations (Figure 1c), while most grains are almost defects free. Furthermore, the dislocations were bowed-out due to pinning at the embedded Y-Ti-enriched nano-oxide particles (Figure 1c) which are distributed in both trans- as well as inter-granular regions. These nanoparticles have a size distribution ranging from 2 to $70 \mathrm{~nm}$ with most exhibiting a diameter below $5 \mathrm{~nm}$.

Upon straining at room temperature, as shown in Figure $1 \mathrm{~b}$, the steel undergoes obvious microstructural changes that include: (1) grain boundary migration which leads to the coarsening of the corresponding grain (compare encircled regions $1 \& 2$ ), (2) grain rotation which leads to its re-orientation with respect to the surrounding grains (compare encircled regions $3 \& 4$ ) and (3) formation of twins (compare encircled regions 5 \& 6). However, as expected for ODS steels, dislocations remained pinned to the embedded Y-Ti-O nanoparticles (Figure 1d). Consequently, in these analyzed regions confined dislocation movement was observed (compare Figure 1d with 1c) which could explain the activation of above mentioned mechanisms.

To understand damage mechanisms, the specimen was strained further until a crack was observed. Figure 2 shows high-angle annular dark-field (HAADF) STEM image and its corresponding orientation map adjacent to the crack. It is evident from Figure $2 b$, that crack propagated dominantly in a transgranular manner. In addition, in some grains the crack is responsible for the splitting of twins (e.g. see marked fractured twins with arrows $1 \& 2$ in Figure $2 \mathrm{~b}$ ). However, it would be interesting to investigate whether the crack propagation is simultaneously accompanied by twin formation.

Room temperature in situ ACOM-TEM straining of novel austenitic ODS steel reveals:

1. Restricted dislocation motion due to pinning of the dislocations at nanoparticles

2. Deformation mechanisms $\rightarrow$ grain boundary migration, grain rotation and twinning

3. Damage mechanisms $\rightarrow$ transgranular crack propagation along with the fracture of twins

\section{References:}

[1] T. Gräning et al, Journal of Nuclear Materials 487 (2017) p. 348.

[2] A. Kobler et al, Ultramicroscopy 128 (2013) p. 68. 


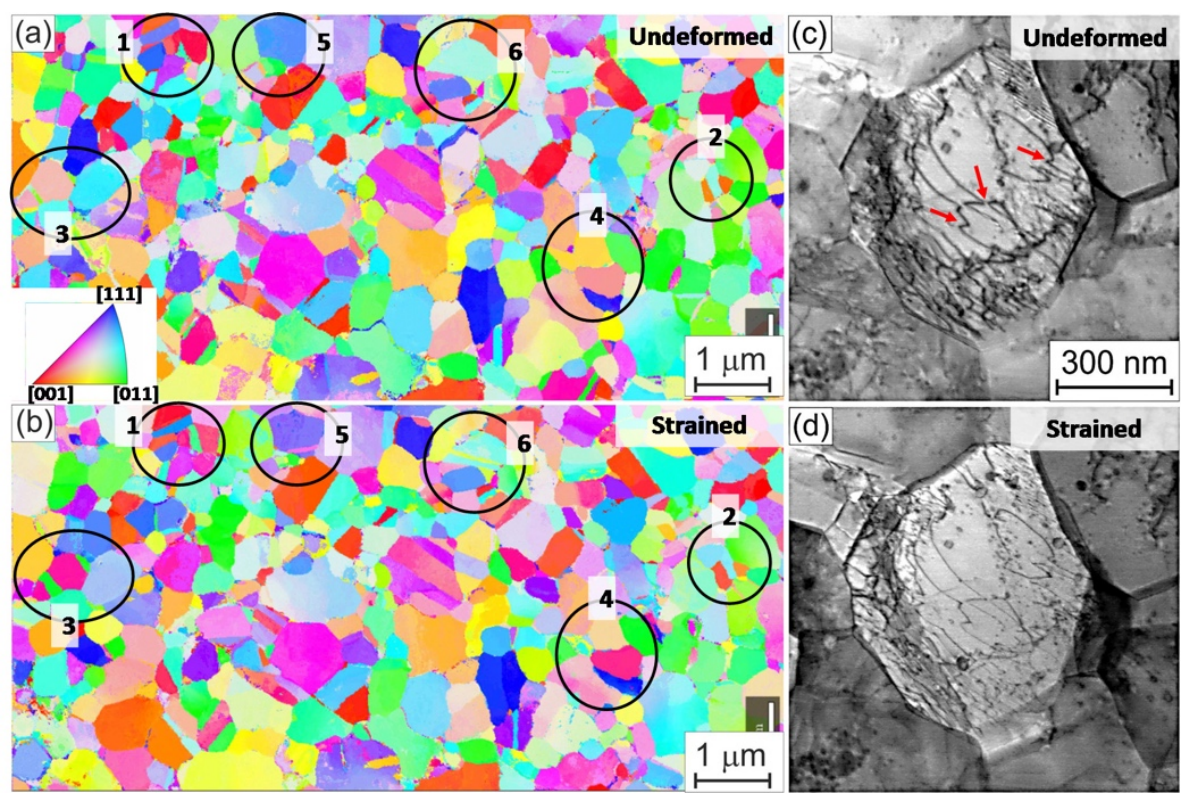

Figure 1. Orientation maps obtained from the same area before (a) and after (b) tensile straining at room temperature. Circles are marked for the comparison of the regions. Inverted contrast HAADF-STEM images show (c) bowed-out dislocations due to pinning (marked) at the Y-Ti-O nanoparticles and (d) confined dislocation motion observed upon straining (compare it with (c)).
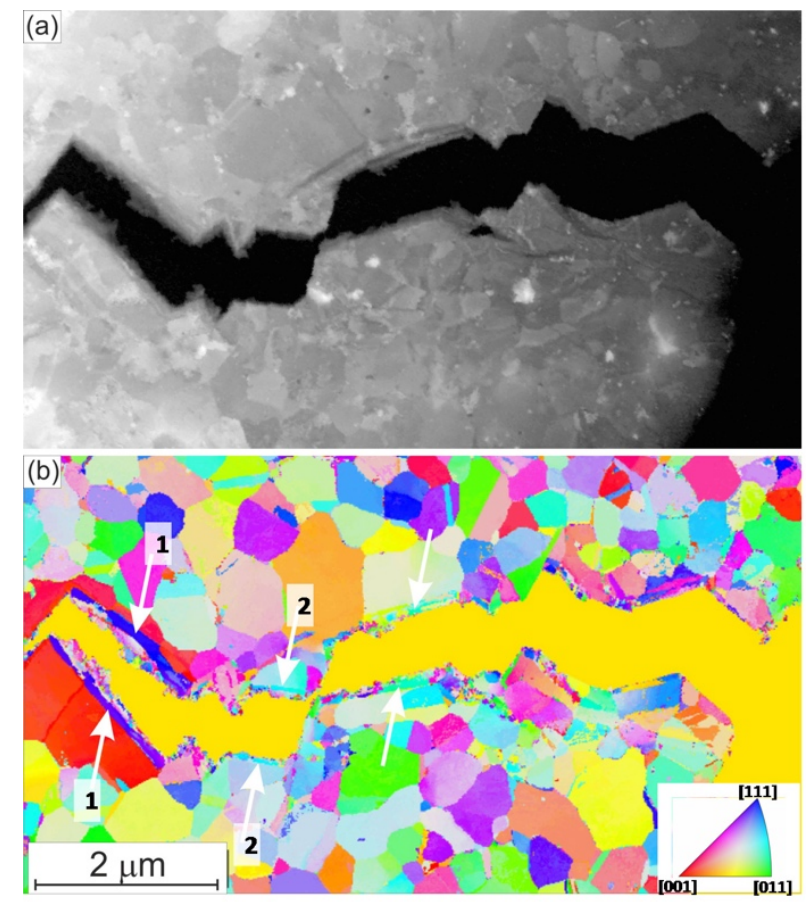

Figure 2. HAADF-STEM image (a) and corresponding orientation map (b) adjacent to the crack. The regions with same orientation separated by crack (yellow area in b) are marked by arrows. Marked arrows $1 \& 2$ show fractured twin. 